\title{
Erratum to: Zero-Sum Pursuit-Evasion Differential Games with Many Objects: Survey of Publications
}

\author{
Sergey S. Kumkov ${ }^{1}$. Stéphane Le Ménec ${ }^{2}$. \\ Valerii S. Patsko 1
}

Published online: 14 December 2016

(C) Springer Science+Business Media New York 2016

\section{Erratum to: Dyn Games Appl (2016) DOI 10.1007/s13235-016-0209-z}

The original version of this article unfortunately contained mistakes in the inline equations of Section 3 and 4. The corrected text has been presented with this erratum.

In the section "Pursuit Problem with Equal Objects Having Simple Motion Dynamics", the extra opening bracket has been removed in the sentence, "Let a feasible control $v(t)$ be given (that is, a measurable function $t \mapsto v(t)$ obeying the constraint $|v(t)| \leq 1$ ).

In the other section "Pursuit Problems with Objects Having General Linear Dynamics", the variable $(t)$ has been removed in the line, "Evolution of the variables $x_{i}, i=\overline{1, k}$, is described as".

The online version of the original article can be found under doi:10.1007/s13235-016-0209-z.

Sergey S. Kumkov

sskumk@gmail.com

Stéphane Le Ménec

stephane.le-menec@mbda-systems.com

Valerii S. Patsko

patsko@imm.uran.ru

1 Krasovskii Institute of Mathematics and Mechanics, Ural Branch of Russian Academy of Sciences, and Institute of Mathematics and Computer Sciences, Ural Federal University,

S. Kovalevskaya str., 16, Ekaterinburg, Russia 620990

2 Airbus Group/MBDA France, 1 Avenue Reaumur, 92358 Le Plessis Robinson Cedex, France 